\title{
转小麦铁蛋白基因酵母的抗氧化活性
}

\author{
赵永亮 $^{1,2}$ 陈 静 $^{1}$ 王 丹 $^{1}$ 王卫国 $^{1}$ 李云辉 $^{1}$
}

${ }^{1}$ 河南工业大学生物工程学院, 河南郑州 $450001 ;{ }^{2}$ 中国农业科学院作物科学研究所 / 农业部品种资源重点实验室 / 农作物基因资源 与基因改良国家重大科学工程, 北京 100081

摘 要: 铁蛋白是广泛存在于动、植物和微生物中的储铁关键蛋白, 具有调节铁代谢平衡、消除亚铁离子引起的氧 化毒性等作用。本研究克隆了小麦铁蛋白基因, 构建其真核表达载体, 经篮选、检测得到具有形成活性小麦铁蛋白多 聚体能力的转基因酵母。转基因酵母与转空载体的对照酵母相比不仅可有效清除过氧化氢 $\left(\mathrm{H}_{2} \mathrm{O}_{2}\right)$, 而且还具清除羟自 由基 $(\cdot \mathrm{OH})$ 和超氧阴离子 $\left(\mathrm{O}_{2}^{-}\right)$的能力; $\cdot \mathrm{OH} 、 \mathrm{O}_{2}^{-}$和 $\mathrm{H}_{2} \mathrm{O}_{2}$ 对转基因酵母的半致死浓度 $\left(\mathrm{LD}_{50}\right)$ 分别为 $0.40 、 1.00$ 和 36.91 $\mathrm{mmol} \mathrm{L}{ }^{-1}$, 比对照酵母分别提高了 $37.93 \% 、 47.06 \%$ 和 $77.03 \%$ 。不同浓度转基因酵母对. $\mathrm{OH} 、 \mathrm{O}_{2}^{-}$和 $\mathrm{H}_{2} \mathrm{O}_{2}$ 清除能力的 回归方程分别为一元三次多项式、对数方程和负指数方程。说明小麦铁蛋白基因的真核表达产物可以有效清除 3 种 活性氧, 而且具有较为复杂的互不相同的作用机制。

关键词: 小麦; 铁蛋白; 转基因酵母; 抗氧化活性

\section{Antioxidative Activities of Transgenic Yeast with Ferritin Gene from Wheat}

\author{
ZHAO Yong-Liang ${ }^{1,2}$, CHEN Jing $^{1}$, WANG Dan ${ }^{1}$, WANG Wei-Guo ${ }^{1}$, and LI Yun-Hui ${ }^{1}$ \\ ${ }^{1}$ Department of Biology Engineering, Henan University of Technology, Zhengzhou 450001, China; ${ }^{2}$ The National Key Facility for Crop Gene Re- \\ sources and Genetic Improvement, Key Laboratory of Crop Germplasm and Biotechnology, Ministry of Agriculture, Institute of Crop Sciences, Chi- \\ nese Academy of Agricultural Sciences, Beijing 100081, China
}

\begin{abstract}
Ferritin, a key protein to store iron, is widely distributed in animal, plant, and microbe. Ferritin can regulate the balance in iron metabolism and decrease the oxidative damage caused by ferrous ion. It is generally recognized that the antioxidant activity of ferritin is mainly caused by integrating ferrous ion among cells, and thereby decreasing the generation of Fenton reaction. In addition to the antioxidant pathway against hydrogen peroxide $\left(\mathrm{H}_{2} \mathrm{O}_{2}\right)$, less information is available on other antioxidative pathways and mechanism of ferritin. In this study, a ferritin gene was cloned from wheat (Triticum aestivum L.), and its expression vehicle of eukaryotic cell was constructed and transformed into yeast (Saccharomyces cerevisiae). Compared with the control yeasts, the transgenic yeasts were able to scavenge $\mathrm{H}_{2} \mathrm{O}_{2}, \mathrm{O}_{2}^{-}$, and $\cdot \mathrm{OH}$ effectively. The half lethal density $\left(\mathrm{LD}_{50}\right)$ of transgenic yeasts induced by the three types of active oxygen $\left(\cdot \mathrm{OH}, \mathrm{O}_{2}^{-}\right.$, and $\left.\mathrm{H}_{2} \mathrm{O}_{2}\right)$ was $0.40,1.00$, and $36.91 \mathrm{mmol} \mathrm{L}^{-1}$, which increased by $37.93 \%, 47.06 \%$, and $77.03 \%$ compared with the control respectively. The abilities for eliminating $\cdot \mathrm{OH}, \mathrm{O}_{2}^{-}$, and $\mathrm{H}_{2} \mathrm{O}_{2}$ of the trsnsgenic yeast per unit concentiation were quantitated with cubic polynomial, logarithmic, and negative exponential equations based on yeast concentration respectively. These results demonstrated that expressed products of wheat ferritin gene in eukaryotic orgamism can effectivly in eliminate various types of active oxygen with different and complex mechanisms.
\end{abstract}

Keywords: Wheat; Ferritin; Trangenic yeast; Antioxdative activity

自由基是机体的正常代谢产物，生物组织中许 多生化反应的中间代谢过程都伴有自由基的产生。 自由基又极易引起化学反应, 可与生物体内的许多 物质如 DNA、脂肪酸、蛋白质等作用, 夺去它们的 氢原子, 造成相关细胞结构与功能的变化, 从而引 起疾病和衰老。生物内总自由基中 $95 \%$ 以上属于氧 自由基(oxygen free radical, OFR)，因此，氧自由基 对生物体危害极大。OFR 包括超氧阴离子自由基 $\left(\mathrm{O}_{2}^{\overline{-}}\right)$, 羟基自由基 $(\cdot \mathrm{OH})$, 过氧化氢 $\left(\mathrm{H}_{2} \mathrm{O}_{2}\right)$ 等 $^{[1]}$ 。生物 体内有两种酶可直接清除活性氧, 即过氧化氢酶可 清除 $\mathrm{H}_{2} \mathrm{O}_{2}$, 超氧化物歧化酶可清除 $\mathrm{O}_{2}^{-}$。 $\cdot \mathrm{OH}$ 活性最 强, 可不加区分地与细胞中的脂类、蛋白质和 DNA

本研究由国家重点基础研究发展计划(973 计划)项目(2004CB117200)和河南工业大学博士基金项目(150216)资助。

第一作者联系方式: E-mail: zylzhao@yahoo.com.cn

Received(收稿日期): 2009-12-17; Accepted(接受日期): 2010-03-19. 


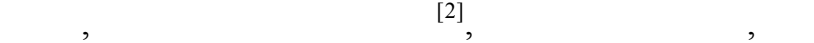
未发现可直接清除. $\mathrm{OH}$ 的酶和抗氧化剂。生物体内 的. $\mathrm{OH}$ 可通过 Fenton 反应 $\left(\mathrm{Fe}^{2+}+\mathrm{H}_{2} \mathrm{O}_{2} \rightarrow \mathrm{Fe}^{3+}+\mathrm{OH}^{-}\right.$ $+\cdot \mathrm{OH})$ 产生。铁是呼吸、光合作用、DNA 合成和新 陈代谢等许多重要生命活动过程中所必需的元素, 因此, 生物体内可溶性铁含量的精密调节十分重 要。由于铁蛋白(Ferritin)具有储铁(将多余活泼的亚 铁离子氧化形成不溶性无毒的生物矿化状态)和放 铁(矿化铁释放)功能, 而成为调节生物体内铁代谢 平衡的关键蛋白 ${ }^{[3]}$ 。Ferritin 广泛存在于动物、植物 和微生物中, 由 24 个亚基构成, 分子量约为 $450 \mathrm{kD}$, 每分子铁蛋白空腔中可储存 4500 个铁原子 ${ }^{[4]}$ 。哺乳 动物铁蛋白由功能互补的重链 $(\mathrm{H})$ 和轻链 $(\mathrm{L})$ 两种亚 基组成, 其分子量分别约为 $28 \mathrm{kD}$ 和 $26 \mathrm{kD}^{[5]}$ 。而 大部分植物铁蛋白只含有一种亚基, 其特性与哺乳 动物的 $\mathrm{H}$ 亚基相近 ${ }^{[6]}$ 。

对过量表达 Ferritin 基因植物的研究表明, 转基 因植株种子中铁含量提高 ${ }^{[7-9]}$, 并且对生物胁迫和非 生物胁迫都有一定的抗性 ${ }^{[10-11]}$ 。转 Ferritin 基因植株 的生物抗性和非生物抗性主要由铁蛋白的抗氧化作 用而引起。目前普遍认为 Ferritin 通过截获细胞间铁 而阻止铁参与 Fenton 反应, 产生具有很强活性 的· $\mathrm{OH}$, 而保护细胞免受因各种环境胁迫而导致的 细胞氧化性损伤 ${ }^{[12]}$ 。比如细胞中铁蛋白的诱导合成 与其抗 $\mathrm{H}_{2} \mathrm{O}_{2}$ 的活性相关 ${ }^{[13]}$; 肿瘤细胞对氧化剂的敏 感性与 Ferritin 基因的表达水平一致 ${ }^{[14-15]}$ 。通过对 Ferritin 基因缺失拟南芥的研究发现, Ferritin 在拟南 芥中的主要作用是防御由自由铁诱导的氧化胁迫, 而非仅仅在发育过程提供铁源 ${ }^{[16]}$ 。上述试验大都是 用 $\mathrm{H}_{2} \mathrm{O}_{2}$ 作为活性氧, Ferritin 是否具有清除其他形式 活性氧的能力及其作用机制未见报道。

作为单细胞真核生物, 酵母多用来表达其他真 核生物的蛋白, 用于蛋白生物活性分析。有学者曾 将人类、蝌蚪等的 Ferritin 基因转入酵母中, 研究其 储铁功能 ${ }^{[17-18]}$, 及其与 Frataxin (铁硫簇相关蛋白)基 因的相互作用 ${ }^{[19]}$ 。但未见将植物的 Ferritin 基因转入 酵母, 并研究其抗氧化活性的报道。本研究以小麦 Ferritin 的 cDNA 序列为目标基因 ${ }^{[20]}$, 以转基因酵母 为平台研究小麦铁蛋白对 3 种活性氧的抗性。

\section{1 材料与方法}

1.1 试验材料

小麦品种中国春由中国农业科学院作物科学研
究所农业部品种资源重点实验室提供。酵母 DY1457 由 Wisconsin 大学 David Eide 博士惠赠。于田间种植 中国春, 花药成熟前去雄, 在授粉后 2 5 d 取未成熟 的籽粒提取总 $\mathrm{RNA}^{[20]}$ 。

\section{2 小麦铁蛋白基因的克隆及表达载体的构建}

用 Superscript II 反转录试剂盒(Invitrogen)将总 RNA 经反转录合成第一链 $\mathrm{cDNA}$ 。以单链 $\mathrm{cDNA}$ 为 模板, 进行 RT-PCR 扩增。分别采用凝胶回收试剂 盒、pGEM-Teasy 连接试剂盒和质粒提取试剂盒(天 根公司)回收、连接转化扩增产物, 并提取质粒。参 照我们先前的报道设计引物、测序等 ${ }^{[20]}$

小麦铁蛋白基因(TaFer)全长编码序列的两端分 别加上 Not I 和 BamH I 接头, 载体 pYES2.0 (Invitrogen)和带接头的 TaFer 序列分别经 Not I 和 BamH I 双酶切后, 用 $\mathrm{T} 4$ 连接酶连接, 形成 pYES2.0-TaFer 重组质粒。重组质粒通过醋酸锂法 ${ }^{[21]}$ 转入野生型酵 母 DY1457 (MAT_ade6 can1 his3 leu2 trp1 ura3), 通 过质粒 PCR 和 DNA 测序检验重组子, 同时将空载 体 pYES2.0 转入 DY1457 中作为对照。将转基因酵 母保存在 YPD培养基上，或者用 $\mathrm{D}$-半乳糖代替葡萄 糖以诱导目标基因的表达。

\subsection{TaFer 在酵母(Sacharomyces cerevisiae)中} 的表达

将转 TaFer 酵母培养在 YP 和 $2 \%$ 半乳糖的液体 培养基中至 $\mathrm{OD}$ 值为 1.0 时，离心收集细胞，并重新 悬浮于 3 倍体积的缓冲液中 $\left(20 \mathrm{mmol} \mathrm{L}^{-1} \mathrm{Tris}-\mathrm{HCl}\right.$, $150 \mathrm{mmol} \mathrm{L}^{-1} \mathrm{NaCl}, 1 \mathrm{mmol} \mathrm{L}{ }^{-1}$ EDTA), 用超声波 (微探头, 强度 $30 \%$ ，每 $30 \mathrm{~s} 10$ 个循环)破碎酵母细 胞, $10000 \times g$ 离心 $10 \mathrm{~min}$ 分离可溶性蛋白与不溶性 蛋白，重组铁蛋白是可溶性蛋白。将可溶性的提取 液 $70^{\circ} \mathrm{C}$ 加热 $10 \mathrm{~min}$, 冰浴 $10 \mathrm{~min}, 10000 \times \mathrm{g}$ 离心 10 $\mathrm{min}$, 除去变性的蛋白质, 进一步纯化重组铁蛋白。 通过考马斯亮蓝染色的 SDS-PAGE 胶来验证部分纯 化的重组铁蛋白单体的分子量大小 ${ }^{[22]}$, 用布鲁士蓝 $\left[2 \% \mathrm{~K}_{4} \mathrm{Fe}(\mathrm{CN})_{6}\right.$ 溶解于 $\left.2 \% \mathrm{HCl}\right]$ 染色的自然 PAGE 胶 验证 24 亚基的具有储铁功能的铁蛋白多聚体 ${ }^{[23]}$ 。采 用 Bradford 法测定蛋白质浓度 ${ }^{[24]}$ 。为比较来自转基 因酵母的重组铁蛋白和未成熟小麦种子中的铁蛋白, 用末成熟小麦种子作为阳性对照。

1.4 转基因酵母对活性氧清除率的测定

1.4.1 羟自由基 $(\cdot \mathrm{OH})$ 采用金鸣等 ${ }^{[25]}$ 的方法测 定转基因酵母对· $\mathrm{OH}$ 的清除率。取 $0.2 \mathrm{~mol} \mathrm{~L}^{-1}(\mathrm{pH} \mathrm{8.4)}$ 的磷酸缓冲液 $2 \mathrm{~mL}$ 放入无菌离心管中，依次加入无 
菌水、 $60 \mathrm{mg} \mathrm{mL}^{-1}$ 菌悬液、 $2 \mathrm{mmol} \mathrm{L}^{-1}$ 硫酸亚铁 $1 \mathrm{~mL}$ 、 $0.1 \mathrm{~mol} \mathrm{~L}^{-1} \mathrm{H}_{2} \mathrm{O}_{2} 1 \mathrm{~mL} 、 2 \mathrm{mmol} \mathrm{L}^{-1}$ 邻二氮菲-无水乙 醇溶液 $1 \mathrm{~mL}$, 总体积为 $7 \mathrm{~mL}$, 加盖, 混匀, $37^{\circ} \mathrm{C}, 180$ $\mathrm{r} \mathrm{min}{ }^{-1}$ 振荡反应 $60 \mathrm{~min}, 5000 \times \mathrm{g}$ 离心 $2 \mathrm{~min}$; 用分光 光度计在 $536 \mathrm{~nm}$ 处测定各管溶液吸光值, 用无菌水 调零。清除率 $=\left(A_{0}-A_{1}\right) \times 100 \% /\left(A_{2}-A_{1}\right)$, 式中 $A_{0}$ 为加 入酵母的. $\mathrm{OH}$ 体系(即 $\mathrm{Fe}^{2+}$-邻二氮菲 $+\mathrm{H}_{2} \mathrm{O}_{2}$ +酵母)的 吸光度; $A_{1}$ 为加入 $\mathrm{H}_{2} \mathrm{O}_{2}$ 的 $\cdot \mathrm{OH}$ 体系(即 $\mathrm{Fe}^{2+}$-邻二氮菲 $+\mathrm{H}_{2} \mathrm{O}_{2}$ ) 的吸光度; $A_{2}$ 为不加入 $\mathrm{H}_{2} \mathrm{O}_{2}$ 的. $\mathrm{OH}$ 体系(即 $\mathrm{Fe}^{2+}$-邻二氮菲溶液)的吸光度。单位浓度转基因酵母 清除. $\mathrm{OH}$ 能力的计算公式为 $\mathrm{Z}=(T-C) / D$, 式中 $\mathrm{Z}$ 表示 单位浓度转 TaFer 酵母与转空载体酵母相比清除率 的增加值, 单位为 $\% \mathrm{mg}^{-1} \mathrm{~mL}^{-1} ; T$ 指一定浓度下转基 因酵母对 $\cdot \mathrm{OH}$ 的清除率; $C$ 为相同浓度下对照酵母 对 $\mathrm{OH}$ 的清除率; $D$ 为酵母浓度, 单位为 $\mathrm{mg} \mathrm{mL}^{-1}$ 。

\subsection{2 超氧阴离子自由基 $\left(\mathrm{O}_{2}^{-}\right)$采用连苯三酚} 自氧化法测定 ${ }^{[26]}$ 转基因酵母对 $\mathrm{O}_{2}^{-}$的清除率。 $15 \mathrm{~mL}$ 无菌离心管中加入 $50 \mathrm{mmol} \mathrm{L}^{-1}(\mathrm{pH}$ 8.2) 的 Tris-HCl 缓冲液 $4.5 \mathrm{~mL}$, 分别加入一定体积的无菌水、 $60 \mathrm{mg}$ $\mathrm{mL}^{-1}$ 菌悬液和 $45 \mathrm{mmol} \mathrm{L}^{-1}$ 邻苯三酚 $0.1 \mathrm{~mL}$, 总体 积为 $7 \mathrm{~mL}$, 混匀; $25^{\circ} \mathrm{C}$ 下 $180 \mathrm{r} \mathrm{min}^{-1}$ 振荡反应 20 $\min ; 5000 \times g$ 离心 $8 \mathrm{~min} ; 420 \mathrm{~nm}$ 处测定溶液吸光值, 用无菌水调零。清除率 $=\left(A_{0}-A_{1}\right) \times 100 \% / A_{0}$, 式中 $A_{0}$ 为末加酵母的反应体系的吸光值, $A_{1}$ 表示加入一定 体积菌悬液的反应体系的吸光值。计算单位浓度转 基因酵母 $\mathrm{O}_{2}^{-}$的清除能力。

1.4.3 过氧化氢 $\left(\mathrm{H}_{2} \mathrm{O}_{2}\right)$ 参照张海容 ${ }^{[27]}$ 的方法测 定转基因酵母对 $\mathrm{H}_{2} \mathrm{O}_{2}$ 的清除率。在 $15 \mathrm{~mL}$ 无菌离心 管中分别加入无菌水、 $60 \mathrm{mg} \mathrm{mL}^{-1}$ 菌悬液和 $0.1 \mathrm{~mol}$ $\mathrm{L}^{-1} \mathrm{H}_{2} \mathrm{O}_{2}$ 溶液 $3.0 \mathrm{~mL}$, 总体积为 $4.0 \mathrm{~mL}$, 混匀; 避光 反应 $60 \mathrm{~min}$, 每 $10 \mathrm{~min}$ 振荡一次; $5000 \times g$ 离心 $2 \mathrm{~min}$; $240 \mathrm{~nm}$ 处测定溶液吸光值, 用蒸馏水调零。清除率 $=\left(A_{0}-A_{1}\right) \times 100 \% / A_{0}$, 式中 $A_{0}$ 为末加酵母的反应体系 的吸光值; $A_{1}$ 表示加入一定体积菌悬液的反应体系 的吸光值。计算单位浓度转基因酵母对 $\mathrm{H}_{2} \mathrm{O}_{2}$ 的清除 能力。

\section{5 致死和半致死浓度的测定}

参照蔡静华等 ${ }^{[28]}$ 的方法测定活性氧对转基因酵 母的致死和半致死浓度。将 $60 \mathrm{mg} \mathrm{mL}^{-1}$ 转基因酵母 菌悬液稀释至 $2000 \sim 3000$ 个菌体 $\mathrm{mL}^{-1}$, 取 $1 \mathrm{~mL}$ 稀 释液, 按照上述方法加入不同浓度的活性氧, $1 \mathrm{~h}$ 后, 取 $1 / 10$ 体积的反应液, 加入含有诱导培养基的平血 中, 用涂布棒涂布均匀, $28^{\circ} \mathrm{C}$ 培养 $48 \mathrm{~h}$, 然后读取菌
落数。致死率 $=\left(C_{0}-C_{1} / C_{0}\right) \times 100 \%$, 式中 $C_{0}$ 表示对照 菌落数; $C_{1}$ 为样品菌落数。根据不同浓度的活性氧对 转基因酵母的致死率，做出相应的线性方程，并计 算出决定系数 $R^{2}$ 、半致死浓度 $L C_{50}$ 和致死率达 $90 \%$ 时的作用浓度 $L C_{90}$ 。

\section{6 统计分析}

所有实验均 3 次重复, 数据以平均值沶准差来 表示。用 Microsoft Excel 2003 进行生物统计, 用 $t$ 检验判别显著性差异。

\section{2 结果与分析}

\section{1 重组小麦铁蛋白在酵母中的表达}

转 TaFer 酵母表达一个近 $28 \mathrm{kD}$ 的蛋白质, 与 天然的小麦 Ferritin 分子量一致, 而转空载体的对照 酵母中没有类似分子量的蛋白质(图 1-A)。因此认为, 过量表达的重组蛋白是由于铁蛋白基因的插入造成 的。铁离子染色的非变性 PAGE 胶中, 能观察到一 条与马脾铁蛋白迁移率一致的条带(图 1-B)。在 SDS 变性 PAGE 胶中的条带揭示的是铁蛋白亚基的情况 (图 1-A), 而自然非变性 PAGE 胶中说明的是完整铁 蛋白多聚体的状态，铁离子染色结果显示培养基中的 铁被酵母菌吸收并且进入重组小麦铁蛋白中(图 1-B),

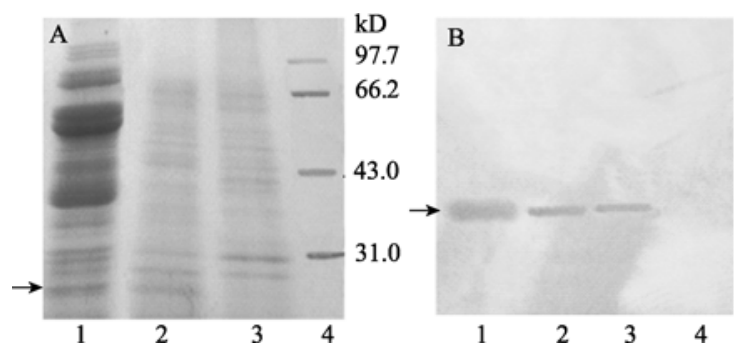

图 1 转基因酵母中重组小麦铁蛋白的表达、分离和铁含量检测 Fig. 1 Expression, isolation and iron content of recombinant TaFer from transformed yeast

A: 转基因酵母表达产物的 SDS-PAGE (12\%聚丙烯酰胺)凝胶电 泳 (考马斯亮蓝染色)。图 A 中, 1: 未成熟小麦种子的细胞提取物; 2: 转 TaFer 酵母的细胞提取物; 3: 转空载体酵母的细胞提取物; 4: 标准蛋白(北京索莱宝预染低分子量蛋白 Marker)。箭头示铁 蛋白亚基。B: 转基因酵母表达产物的自然 PAGE (5\%聚丙烯酰 胺)凝胶电泳(普鲁士蓝染色)。图 B 中 1：马脾铁蛋白; 2 : 未成熟 小麦种子的细胞提取物; 3: 转 TaFer 酵母的细胞提取物; 4: 为转空 载体酵母的细胞提取物。箭头示完整有储铁功能的铁蛋白聚合物。

A: SDS-PAGE ( $12 \%$ polyacrylamide gel) of extracts after partial purification stained by Coomassie blue. In panel A, 1: immature wheat seed extract; 2: yeast/pYES2.0-TaFer cell extract; 3: yeast/pYES2.0 cell extract; 4: protein marker. Ferritin subunits are marked with an arrow. B: Native PAGE (5\% polyacrylamide gel) of protein extract stained with Prussian blue. In Panel B, 1: horse spleen Ferritin; 2: immature wheat seed extract; 3: yeast/YES2.0aFer cell extract; 4: yeast/pYES2.0 cell extract. Intact Ferritin is marked with an arrow. 
从而全面说明转基因酵母中的铁蛋白基因正确表达, 而且有效组装成为有功能的铁蛋白多聚体分子。

\section{2 转 TaFer 酵母对. OH 的抗性}

转空载体酵母和转 $\mathrm{TaFer}$ 酵母对· $\mathrm{OH}$ 都有一定 的抗性(图 2-A)。但是转基因酵母与对照相比具有更 强的抗 $\mathrm{OH}$ 的活性。在低酵母浓度时 $\left(2.5 \mathrm{mg} \mathrm{mL}{ }^{-1}\right)$, 它们对. $\mathrm{OH}$ 的清除能力的差异并不明显, 但是随着 浓度的增加, 这种差异变得越来越显著。当酵母浓 度为 $10 \mathrm{mg} \mathrm{mL}$-1 时, 单位浓度转基因酵母清除 $\cdot \mathrm{OH}$ 的能力最强, 为 $1.48 \% \mathrm{mg}^{-1} \mathrm{~mL}^{-1}$; 而当酵母浓度为 $2.5 \mathrm{mg} \mathrm{mL} \mathrm{m}^{-1}$ 时, 该值最小, 为 0.73 , 最大值与最小 值相差 1.03 倍(图 2-B), 说明酵母浓度显著影响其对 $\cdot \mathrm{OH}$ 的清除。酵母浓度较低 $\left(2.5 \sim 5.0 \mathrm{mg} \mathrm{mL}^{-1}\right)$ 和较高 (15.0 17.5 mg mL ${ }^{-1}$ )时, 单位浓度的转基因酵母对 . $\mathrm{OH}$ 清除力较低, 而在酵母浓度适中(7.5 12.5 mg
$\mathrm{mL}^{-1}$ )时, 该值较高(图 2-B), 说明酵母浓度过低和过 高都不利于转基因酵母对· $\mathrm{OH}$ 的清除。不同酵母浓 度 $(x)$ 与单位浓度转基因酵母对. $\mathrm{OH}$ 清除能力 $(y)$ 的回 归方程为 $y=0.0066 x^{3}-0.1428 x^{2}+0.8201 x+0.0565$, $R^{2}=0.9948$ 。

\section{3 转 TaFer 酵母对 $\mathrm{O}_{2}^{-}$的抗性}

无论是对照还是转基因酵母对 $\mathrm{O}_{2}^{-}$都有一定的 抗性, 但是转基因酵母与对照相比具有更强的抗 $\mathrm{O}_{2}^{-}$ 的活性, 即使在酵母低浓度时 $\left(1.0 \mathrm{mg} \mathrm{mL}{ }^{-1}\right)$ 对 $\mathrm{O}_{2}^{-}$的 清除能力即有显著差异, 随着浓度的增加, 这种差 异越来越显著(图 2-C)。当酵母浓度为 $1.0 \mathrm{mg} \mathrm{mL}^{-1}$ 时, 单位浓度转基因酵母清除 $\mathrm{O}_{2}^{-}$的能力最强, 为 $9.37 \% \mathrm{mg}^{-1} \mathrm{~mL}^{-1}$; 而当酵母浓度为 $17.50 \mathrm{mg} \mathrm{mL}^{-1}$ 时, 该值最小, 为 $2.0 \% \mathrm{mg}^{-1} \mathrm{~mL}^{-1}$, 最大值与最小值
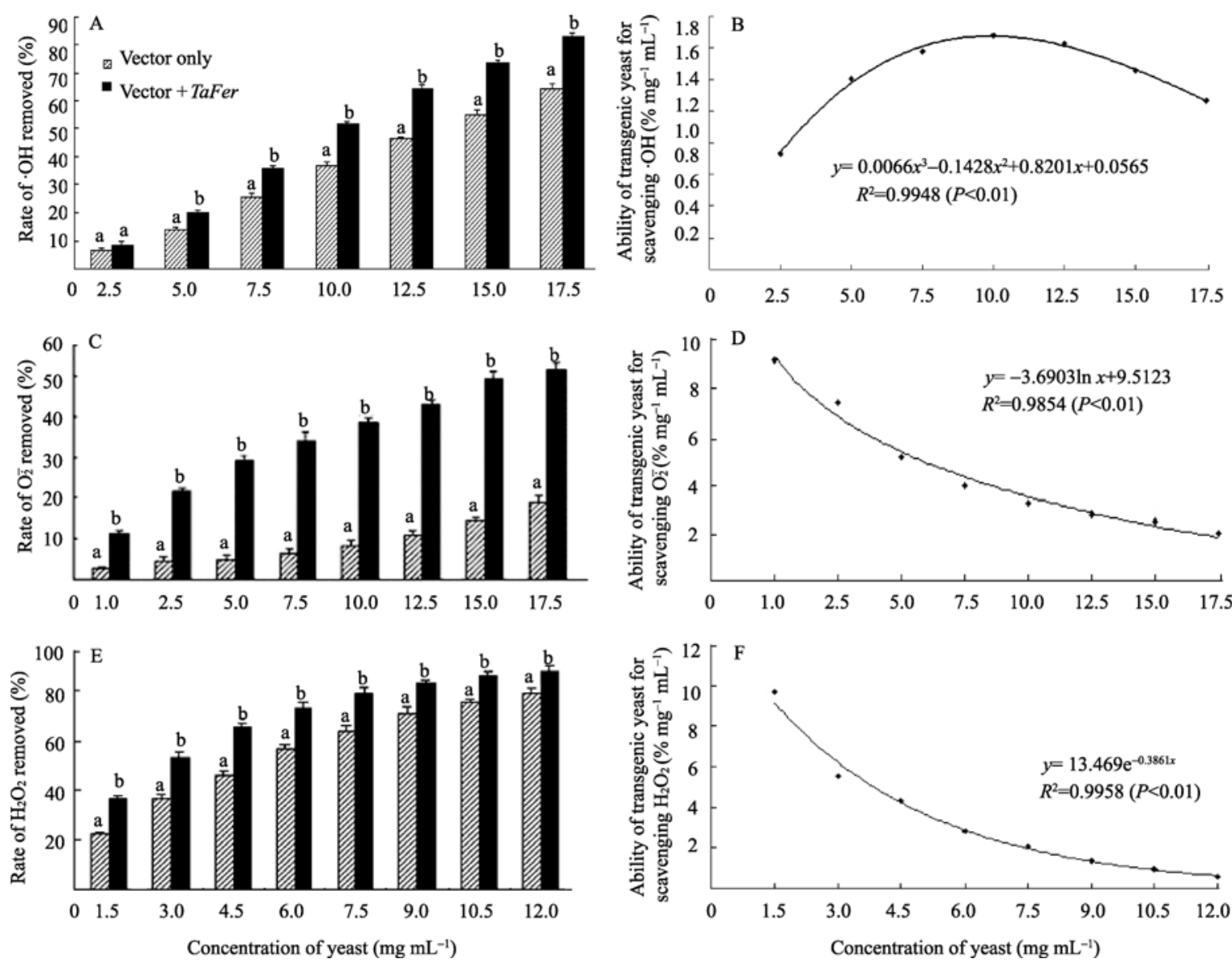

图 2 转基因酵母对活性氧的清除能力

Fig. 2 Characteristics of eliminating active oxygen of transgenic yeasts

转基因酵母在由半乳糖诱导的 YP 液体培养基中生长过夜, 用双蒸水清洗细胞并调至特定浓度, 用高浓度的活性氧 $\left(\cdot \mathrm{OH} 、 \mathrm{O}_{2}^{\overline{2}} 、 \mathrm{H}_{2} \mathrm{O}_{2}\right)$ 分别处理转基因酵母, 并测定清除率, 数据为 3 次独立平行实验的平均值 \pm 标准差, 不同小写字母表示转基因酵母和对照对活性氧清 除率差异显著 $(P<0.05)$ 。

Transformed strains were grown overnight in liquid YP and galactose medium. The cells were adjusted to the concentration indicated. After treated with high concentrations of $\cdot \mathrm{OH}, \mathrm{O}_{2}^{-}$, and $\mathrm{H}_{2} \mathrm{O}_{2}$, the decrease rates were measured. The values are mean $\pm S D$ of three independent experiments. Bars marked with different letters are significantly different between transgenic yeasts and control at $P<0.05$. 
相差 3.7 倍, 说明转基因酵母浓度显著影响其对 $\mathrm{O}_{2}^{-}$ 的清除。从图 2-D 还可以看出酵母浓度最低时, 单 位浓度的转基因酵母对 $\mathrm{O}_{2}^{-}$的清除能力最强, 而在酵 母浓度最高时, 该值最低, 说明高浓度酵母不利于 转基因酵母对 $\mathrm{O}_{2}^{-}$的清除。不同酵母浓度 $(x)$ 与单位浓 度转小麦铁蛋白基因酵母对 $\mathrm{O}_{2}^{-}$清除能力 $(y)$ 的回归 方程为 $y=-3.6903 \ln x+9.5123, R^{2}=0.9854$ 。

\section{4 转小麦铁蛋白酵母对 $\mathrm{H}_{2} \mathrm{O}_{2}$ 的抗性}

对照和转基因酵母对 $\mathrm{H}_{2} \mathrm{O}_{2}$ 都有一定的清除能 力, 但是转基因酵母与对照相比具有更强的清除 $\mathrm{H}_{2} \mathrm{O}_{2}$ 的活性, 其差异达显著水平(图 2-E)。当酵母浓 度为 $1.5 \mathrm{mg} \mathrm{mL}^{-1}$ 时, 单位浓度转基因酵母清除 $\mathrm{H}_{2} \mathrm{O}_{2}$ 的能力最强, 为 $9.7 \% \mathrm{mg}^{-1} \mathrm{~mL}^{-1}$; 而当酵母浓 度为 $12.0 \mathrm{mg} \mathrm{mL}^{-1}$ 时, 该值最小, 为 $0.6 \% \mathrm{mg}^{-1} \mathrm{~mL}^{-1}$, 最大值与最小值相差 15.1 倍, 说明转基因酵母浓度 极显著地影响其对 $\mathrm{H}_{2} \mathrm{O}_{2}$ 的清除。酵母浓度最低时, 单位浓度的转基因酵母对 $\mathrm{H}_{2} \mathrm{O}_{2}$ 清除能力最大, 而酵 母浓度最高时, 该值最低(图 2-F), 说明酵母浓度过 高不利于转基因酵母对 $\mathrm{H}_{2} \mathrm{O}_{2}$ 的清除。不同酵母浓度 $(x)$ 与单位浓度转基因酵母清除 $\mathrm{H}_{2} \mathrm{O}_{2}$ 能力 $(y)$ 的回归 方程为 $y=13.469 \mathrm{e}^{-0.3861 x}, R^{2}=0.9958$ 。

2.53 种活性氧对转基因酵母的半致死浓度和致 死浓度

$\cdot \mathrm{OH}$ 对转 TaFer 基因酵母的 $\mathrm{LD}_{50}$ 和 $\mathrm{LD}_{90}$ 分别为 $0.40 \mathrm{mmol} \mathrm{L}^{-1}$ 和 $0.72 \mathrm{mmol} \mathrm{L}^{-1}$, 而对照酵母的 $\mathrm{LD}_{50}$ 和 $\mathrm{LD}_{90}$ 分别为 $0.29 \mathrm{mmol} \mathrm{L}^{-1}$ 和 $0.53 \mathrm{mmol} \mathrm{L}^{-1}$, 转基 因酵母和对照酵母相比对. $\mathrm{OH}$ 的耐受度分别增加了 $37.93 \%$ 和 35.85\% (图 3-A 和表 1)。

$\mathrm{O}_{2}^{-}$对转基因酵母的 $\mathrm{LD}_{50}$ 和 $\mathrm{LD}_{90}$ 分别为 1.00 $\mathrm{mmol} \mathrm{L} \mathrm{L}^{-1}$ 和 $1.79 \mathrm{mmol} \mathrm{L}^{-1}$ (图 3-B 和表 1), 比对照 $\left(0.68 \mathrm{mmol} \mathrm{L}^{-1}\right.$ 和 $\left.1.24 \mathrm{mmol} \mathrm{L}^{-1}\right)$ 对 $\mathrm{O}_{2}^{-}$的耐受度分别 增加了 $47.06 \%$ 和 $44.35 \%$ 。

$\mathrm{H}_{2} \mathrm{O}_{2}$ 对转基因酵母和对照的 $\mathrm{LD}_{50}$ 和 $\mathrm{LD}_{90}$ 分别 为 $36.91 \mathrm{mmol} \mathrm{L}^{-1} 、 67.42 \mathrm{mmol} \mathrm{L}^{-1}$ 和 $20.58 \mathrm{mmol} \mathrm{L}^{-1}$ 、 $30.05 \mathrm{mmol} \mathrm{L}^{-1}$ (图 3-C 和表 1), 转基因酵母对 $\mathrm{H}_{2} \mathrm{O}_{2}$ 的耐受度分别比对照增加了 $77.03 \%$ 和 $77.18 \%$ 。

\section{3 讨论}

3.1 转基因酵母对 3 种活性氧的清除能力和耐受性 酵母虽然是结构最简单的真核生物, 但其细胞 内含有多种具有抗氧化作用的多糖 ${ }^{[29]}$ 、酶类 ${ }^{[30]}$ 、金 属硫蛋白等物质 ${ }^{[31]}$ ，其提取物有一定的抗氧化活
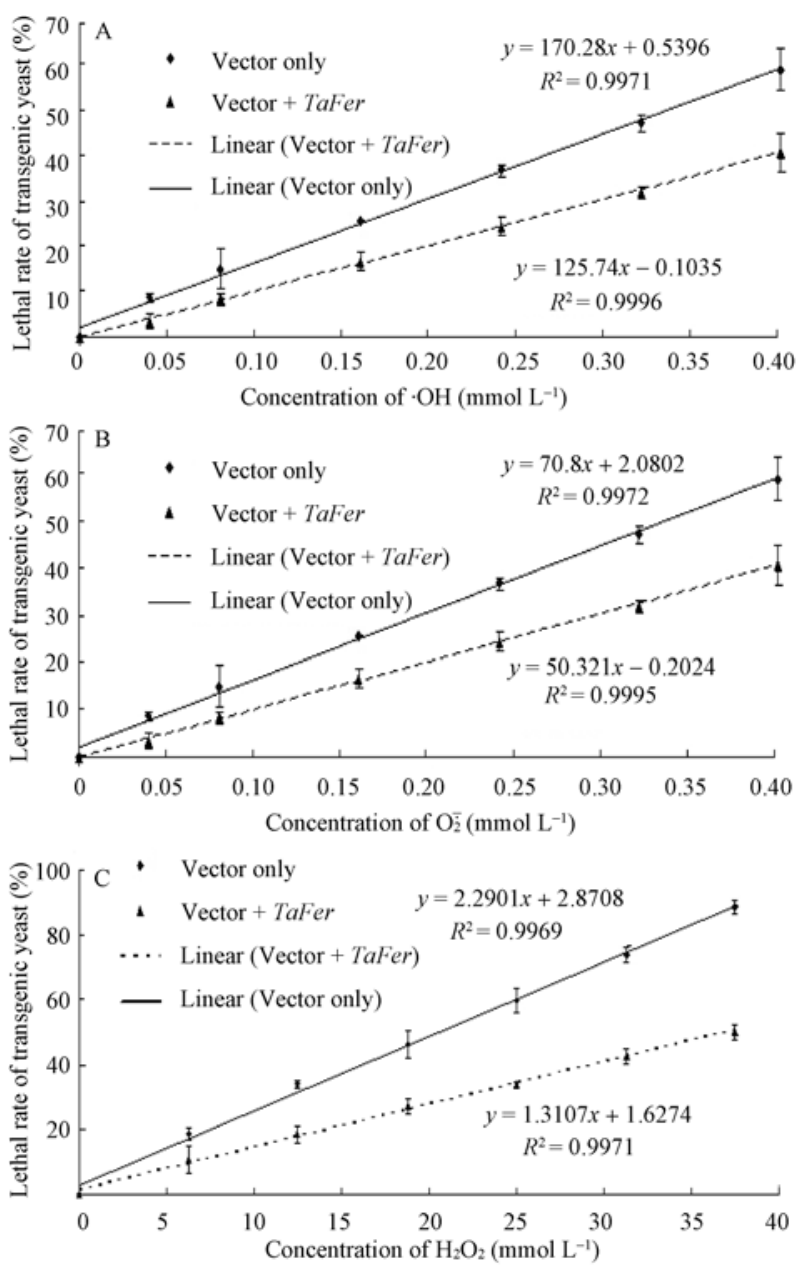

图 33 种活性氧对转基因酵母的致死率

Fig. 3 Lethal rate of transformed strains caused by active oxygens

A: · $\mathrm{OH}$ 对转基因酵母的致死率; $\mathrm{B}$ : $\mathrm{O}_{2}^{-}$对转基因酵母的致死率; C: $\mathrm{H}_{2} \mathrm{O}_{2}$ 对转基因酵母的致死率。转基因酵母在由半乳糖诱导的 YP 培养基中生长过夜, 并调至合适浓度, 分别用不同浓度的活 性氧 $\left(\cdot \mathrm{OH}, \mathrm{O}_{2}{ }^{-}, \mathrm{H}_{2} \mathrm{O}_{2}\right)$ 处理 $60 \mathrm{~min}$, 涂平板, $28^{\circ} \mathrm{C}$ 培养 $48 \mathrm{~h}$, 观察 菌落数; 与对照相比, 求出活性氧对转基因酵母的致死率。图中 数据为 3 次平行实验的平均值 \pm 标准差。

A: lethal rate of transformed strains caused by $\cdot \mathrm{OH}$; B: lethal rate of transformed strains caused by $\mathrm{O}_{2}^{-}$; $\mathrm{C}$ : lethal rate of transformed strains caused by $\mathrm{H}_{2} \mathrm{O}_{2}$. Transformed strains were grown overnight in liquid YP medium induced by galactose. Cells were adjusted to the appropriate concentration and treated with a series of concentrations of $\mathrm{OH}, \mathrm{O}_{2}^{-}$, and $\mathrm{H}_{2} \mathrm{O}_{2}$ for $1 \mathrm{~h}$, respectively. The plates were incubated at $28^{\circ} \mathrm{C}$ for $2 \mathrm{~d}$ before counted clones. The lethal rate was computed through comparison of the clone numbers between control and treated experiments. The values are means $\pm S D$ of three independent experiments.

性 ${ }^{[32]}$ 。本研究也发现转空载体酵母对活性氧有一定 清除能力。但是 TaFer 酵母对活性氧的清除能力显 著高于转空载体酵母, 说明 TaFer 的导入和表达能 够显著提高转基因酵母对 3 种活性氧的清除能力。

转 TaFer 酵母对 3 种活性氧的半致死浓度以 
表 $1 \cdot \mathrm{OH} 、 \mathrm{O}_{2}^{-}$和 $\mathrm{H}_{2} \mathrm{O}_{2}$ 对转基因酵母的半致死浓度 $\left(\mathrm{LD}_{50}\right)$ 和致死浓度 $\left(\mathrm{LD}_{90}\right)$

Table 1 Half lethal density $\left(\mathrm{LD}_{50}\right)$ and lethal density $\left(\mathrm{LD}_{90}\right)$ of transgenic yeasts caused by $\cdot \mathrm{OH}, \mathrm{O}_{2}^{-}$, and $\mathrm{H}_{2} \mathrm{O}_{2}\left(\mathrm{mmol} \mathrm{L}^{-1}\right)$

\begin{tabular}{|c|c|c|c|c|c|c|c|c|c|}
\hline & \multicolumn{3}{|c|}{$\cdot \mathrm{OH}$} & \multicolumn{3}{|c|}{$\mathrm{O}_{2}^{-}$} & \multicolumn{3}{|c|}{$\mathrm{H}_{2} \mathrm{O}_{2}$} \\
\hline & 方程 Equation & $\mathrm{LD}_{50}$ & $\mathrm{LD}_{90}$ & 方程 Equation & $\mathrm{LD}_{50}$ & $\mathrm{LD}_{90}$ & 方程 Equation & $\mathrm{LD}_{50}$ & $\mathrm{LD}_{90}$ \\
\hline $\begin{array}{c}\text { 转空载体 } \\
\text { Vector only }\end{array}$ & $y=170.28 x+0.5396$ & 0.29 & 0.53 & $y=70.8 x+2.0802$ & 0.68 & 1.24 & $y=2.2901 x+2.8708$ & 20.58 & 38.05 \\
\hline $\begin{array}{c}\text { 转 TaFer } \\
\text { Vector }+ \text { TaFer }\end{array}$ & $y=125.74 x-0.1035$ & 0.40 & 0.72 & $y=50.321 x-0.2024$ & 1.00 & 1.79 & $y=1.3107 x+1.6274$ & 36.91 & 67.4 \\
\hline $\begin{array}{c}\text { 耐受性增加 } \\
\text { Tolerance increased (\%) }\end{array}$ & & 37.9 & 35.9 & & 47.1 & 44.4 & & 77.0 & 77.2 \\
\hline
\end{tabular}

$\mathrm{H}_{2} \mathrm{O}_{2}$ 最高; 而且耐受 $\cdot \mathrm{OH} 、 \mathrm{O}_{2}^{-} 、 \mathrm{H}_{2} \mathrm{O}_{2}$ 的半致死浓度 分别比对照提高 $37.9 \%$ 、47.1\%和 $77.0 \%$, 同样也以 $\mathrm{H}_{2} \mathrm{O}_{2}$ 的耐受力增加最多。说明无论是转基因酵母还 是对照酵母对 $\mathrm{H}_{2} \mathrm{O}_{2}$ 的耐受能力远远大于其他两种 活性氧, 而且转基因酵母对 $\mathrm{H}_{2} \mathrm{O}_{2}$ 的清除能力也是最 强的, 这一结果符合已报道的铁蛋白抗氧化作用机 制 ${ }^{[12]}$ 。

\section{2 转小麦铁蛋白基因酵母清除自由基机制}

为了清楚地说明小麦铁蛋白基因的转入对 3 种 活性氧的清除能力, 消除酵母浓度和酵母自身对活 性氧清除能力的影响, 本研究将得到的转基因酵母 对活性氧清除率的数据进行处理, 以单位浓度清除 率的增加值为纵坐标, 酵母浓度为横坐标做图, 得 到不同浓度下单位浓度转基因酵母对活性氧清除能 力的关系图, 并构建了回归方程(图 2-B、D、F), 发 现单位浓度转基因酵母对 3 种活性氧清除能力与自 身浓度之间呈现出不同的关系。对· $\mathrm{OH}$ 来说, 其回归 方程是一元三次多项式, 适中的酵母浓度有利于转 基因酵母对 $\cdot \mathrm{OH}$ 的清除; 对 $\mathrm{O}_{2}^{-}$来说, 其回归方程为 对数方程, 低浓度的转基因酵母有利于其对 $\mathrm{O}_{2}{ }^{-}$的 清除; 对于 $\mathrm{H}_{2} \mathrm{O}_{2}$ 来说, 其回归方程符合负指数方程, 低浓度的转基因酵母有利于其对 $\mathrm{H}_{2} \mathrm{O}_{2}$ 的清除。不同 的回归方程反映数据之间不同的内在关系, 转基因 酵母对 3 种活性氧清除能力形成 3 种不同的回归方 程, 说明铁蛋白对 3 种活性氧的清除存在不同的机 制, 而且 3 种回归方程都是非线性复杂方程, 说明小 麦铁蛋白清除活性氧是一个多因素影响的过程。为 揭示影响铁蛋白抗氧化活性的因素和作用机制, 需 要进一步简化作用体系, 而对纯化铁蛋白的抗氧化 活性进行深入研究, 可能有助于问题的解决。

\section{4 结论}

转 TaFer 酵母可以有效地清除 3 种活性氧, $\cdot \mathrm{OH}$ 、 $\mathrm{O}_{2}^{-}$和 $\mathrm{H}_{2} \mathrm{O}_{2}$ 对转基因酵母的半致死浓度比对照分别
提高了 $37.93 \%$ 、47.06\%和 $77.03 \%$ 。不同浓度转基因 酵母对. $\mathrm{OH} 、 \mathrm{O}_{2}^{-}$和 $\mathrm{H}_{2} \mathrm{O}_{2}$ 清除能力的回归方程分别为 一元三次多项式、对数方程和负指数方程。说明小 麦铁蛋白基因的真核表达产物对 3 种活性氧的清除 具有较为复杂的互不相同的作用机制。

\section{References}

[1] Zheng R-L(郑荣梁), Huang Z-Y(黄中洋). Free Radical Biology (自由基生物学), 3rd edn. Beijing: Higher Education Press, 2007. pp 1-25 (in Chinese)

[2] Blokhina O, Virolatinen E, Kurt V F. Antioxidants, oxidative damage and oxygen deprivation stress: a review. Ann Bot, 2003, 91: 179-194

[3] Theil E C, Matzapetakis M, Liu X. Ferritins: Iron/oxygen biominerals in protein nanocages. J Biol Inorg Chem, 2006, 11, 803-810

[4] Harrison P M, Arosio P. Ferritins: molecular properties, iron storage function and cellular regulation. Biochim Biophys Acta, 1996, 1275: 161-203

[5] Theil E C. Ferritin: structure, gene regulation, and cellular function in animals, plants, and microorganisms. Annu Rev Biochem, 1987, 56: 289-315

[6] Wade V J, Treffry A, Laulhere J P, Bauminger E R, Cleton M I, Mann S, Briat J F, Harrison P M. Structure and composition of ferritin cores from pea seed (Pisum sativum). Biochim Biophys Acta, 1993, 1161: 91-96

[7] Goto F, Yoshihara T, Saiki H, Takaiwa F. Iron accumulation and enhanced growth in transgenic lettuce plants expressing the iron-binding protein ferritin. Theor Appl Genet, 2000, 100: 658-664

[8] Ye X(叶霞), Huang X-D(黄晓德), Yao Q-H(姚泉洪), Zhang Z(张振). Agrobacterium-mediated transformation of tomato with PvFer. Acta Hort Sin (园艺学报), 2007, 34(2): 489-492 (in Chinese with English abstract)

[9] Ye H X, Li M, Guo Z J. Evaluation and application of two high-iron transgenic rice lines expressing a pea ferritin gene. Rice Sci, 2008, 15: 51-56

[10] Deak M, Horvath G V, Davletova S, Torok K, Sass L, Vass I, Barna B, Kiraly Z, Dudits D. Plants ectopically expressing the iron-binding protein, ferritin, are tolerant to oxidative damage 
and pathogens. Nat Biotechnol, 1999, 17: 192-196

[11] Hegedus A, Janda T, Horváth G V, Dudits D. Accumulation of overproduced ferritin in the chloroplast provides protection against photoinhibition induced by low temperature in tobacco plants. J Plant Physiol, 2008, 165: 1647-1651

[12] Halliwell B, Gutteridge J M. Protection against oxidants in biological systems: the superoxide theory of oxygen toxicity. In: Free Radicals in Biology and Medicine. New York: Oxford University Press, 1989. pp 86-179

[13] Balla G, Jacob H S, Balla J, Rosenberg M, Nath K, Apple F, Eaton J W, Vercellotti G M. Ferritin: a cytoprotective antioxidant strategem of endothelium. J Biol Chem, 1992, 267: 18148-18153

[14] Baldi A, Lombardi D, Russo P, Palescandolo E, Luca A D, Santini D, Baldi F, Rossiello L, Dellanna M L, Mastrofrancesco A, Maresca V, Flori E, Natali P G, Picardo Mauro, Pagg M G. Ferritin contributes to melanoma progression by modulating cell growth and sensitivity to oxidative stress. Clin Cancer Res, 2005, 11: 3175-3183

[15] Pham C G, Bubici C, Zazzeroni F, Papa S, Jones J, Alvarez K, Jayawardena S, Smaele D E, Cong R, Beaumont C. Ferritin heavy chain upregulation by NF-kappaB inhibits TNF alphainduced apoptosis by suppressing reactive oxygen species. Cell, 2004, 119: 529-542

[16] Ravet K, Touraine B, Boucherez J, Briat J F, Gaymard F, Cellier F. Ferritins control interaction between iron homeostasis and oxidative stress in Arabidopsis. Plant J, 2009, 57: 400-412

[17] Kim H J, Kim H M, Kim J H, Ryu K S, Park S M, Jahng K Y, Yang M K, Kim D H. Expression of heteropolymeric ferritin improves iron storage in Saccharomyces cerevisiae. Appl Environ Microbiol, 2003, 69: 1999-2005

[18] Shin Y M, Kwon T H, Kim K S, Chae K S, Kim D H, Kim J H, Yang M S. Enhanced iron uptake of Saccharomyces cerevisiae by heterologous expression of a tadpole ferritin gene. Appl Environ Microbiol, 2001, 67: 1280-1283

[19] Campanella A, Isaya G, O’Neill H A, Santambrogio P, Cozzi A, Arosio P, Sonia L. The expression of human mitochondrial ferritin rescues respiratory function in frataxin-deficient yeast. $\mathrm{Hu}$ man Mol Genet, 2004, 13: 2279-2288

[20] Zhao Y-L(赵永亮), Zhou R-H(周荣华), Jia J-Z(贾继增). The homologous cloning of the ferritin gene from wheat. $J$ Triticeae Crops (麦类作物学报), 2008, 28(1): 25-30 (in Chinese with English abstract)

[21] Schiestl R H, Gietz R D. High efficiency transformation of intact yeast cells using single stranded DNA as a carrier. Curr Genet, 1989, 16: 339-346

[22] Laemmli U K. Cleavage of structural proteins during the assembly of the head of bacteriophage T4. Nature, 1970, 227: 680-685

[23] Barcelo F, Arean C O, Moore G R. Isolation and preliminary characterization of ferritin from clover seeds. BioMetals, 1995, 8: $47-52$

[24] Bradford M M. Rapid and sensitive method for quantitation of microgram quantities of protein utilizing principle of protein-dye binding. Anal Biochem, 1976, 72: 248-254

[25] Jin M(金鸣), Cai Y-X(蔡亚欣), Li J-R(李金荣), Zhao H(赵辉). 1,10-phenanthroline- $\mathrm{Fe}^{2+}$ oxidative assay of hydroxyl radical produced by $\mathrm{H}_{2} \mathrm{O}_{2} / \mathrm{Fe}^{2+}$. Prog Biochem Biophys (生物化学与生 物物理进展), 1996, 23(6): 553-555 (in Chinese with English abstract)

[26] Marlund S, Marlund G. Involvement of superoxide anion radicals in the autoxidation of pyrogallol and a convininent assay for superoxide dismutase. Eur J Biochem, 1974, 47: 469-471

[27] Zhang H-R(张海容). Study of the scavenging radical activities of polysaccharide of hippophae rhamnosides fruit peel. Bull Bot (植 物学通报), 2005, 22(6): 703-707 (in Chinese with English abstract)

[28] Cai H-J(蔡华静), Xiong Z-Q(熊智强), Tu G-Q(涂国全). A preliminary study on the antibacterial action of the bioactive compound produced by streptomyces 702. Acta Agric Univ Jiangxiensis (江西农业大学学报), 2005, 27(2): 274-278 (in Chinese with English abstract)

[29] Wang Y-H(王义华), Xu M-Z(徐梅珍), Jiang P(江萍), He Z-F(何 照范), Xiong L-Y(熊绿芸). Purification, chemical component and antioxidative analysis of polysaccharide from Saccharomyces cerevisiae. Microbiology (微生物学通报), 2003, 30(4): 51-53 (in Chinese with English abstract)

[30] Sheng W(盛玮). Study on glutathione peroxidese activity in yeast. J Huaibei Ind Teachers Coll (淮北煤炭师范学院学报), 2003, 24(1): 40-43 (in Chinese with English abstract)

[31] Lin Z-L(林稚兰), Hao F-Y(郝福英), Wang L(王龙), Jia L(贾乐), Tian F(田枫), Li Q(李奇). Study on antioxidation of Saccharomyces cerevisiae Cu-metallothionein. Mycosystema (菌物学报), 1997, 15(4): 291-296 (in Chinese with English abstract)

[32] Zhang K-S(张坤生), Zhang L(张亮), Lü X-L(吕晓玲), Chen X-J(陈晓娟). The study on antioxidant activity of Rhodotorular glutinis pigment. Food Sci (食品科学), 2004, 25(3): 61-63 (in Chinese with English abstract) 